hep-ph/0003227

\title{
New Physics Effects from B Meson Decays 1
}

\author{
Anirban Kundu \\ E-mail: akundu@juphys.ernet.in \\ Department of Physics, Jadavpur University, Calcutta - 700032, India
}

\begin{abstract}
In this talk, we point out some of the present and future possible signatures of physics beyond the Standard Model from B-meson decays, taking R-parity conserving and violating supersymmetry as illustrative examples.
\end{abstract}

\section{Introduction}

It has long been established that the B-meson system (both charged and neutral) may be the ideal place to look for indirect effects of physics, both CP-conserving and CP-violating, beyond the Standard Model (BSM) [1]. The reasons, in brief, are:

- The $B-\bar{B}$ mixing is dominated by the short-distance box diagram with the top quark running inside the loop. Thus, CP-violation is large and the major part of it, fortunately, can be calculated to a good precision. The long-distance part is anyway known to be negligible in B-decays (this may be compared with $\mathrm{D}$ decays where it is the dominant contribution).

- The soft QCD effects are less pronounced for B than for D - this is due to the $m_{c} / m_{b}$ suppression. Thus, $1 / m_{b}$ corrections are at least controllable.

- Due to the CKM suppression, the lifetime of the B meson is sufficiently large to be accurately measured. This has important implications in the asymmetric B-factories.

- Due to the abovementioned reasons, we will have a number of dedicated B-factories in the near future, apart from the running ones like CLEO. We will need the hadronic machines to measure $\mathrm{CP}$-violation in modes with branching ratios (BR) less than $10^{-5}$.

However, before one proceeds, one must remember that the theoretical uncertainties are still significant, and will probably remain so in near future [2]. These uncertainties are dominated by our ignorance of soft-QCD physics. For example, approximations like factorization and quark-hadron duality (both local and global) are not at all beyond doubt; the numerical inputs like the strange quark mass, the number of effective colour $N_{c}$ and the regularization scale $\mu$ are not yet certain and should be treated as more or less free parameters. With all these handicaps, it is extremely difficult to find the signature of BSM physics if that is more than one order of magnitude smaller than the SM contribution.

Fortunately, there are cases when the BSM signal may be equally (or more) large as the $\mathrm{SM}$ one, and can be easily distinguished. There are two major ways to proceed. First, one can

\footnotetext{
${ }^{1}$ Talk presented at the Sixth Workshop on High Energy Particle Phenomenology (WHEPP-6), Chennai, India
} 
look for CP-asymmetries, both direct and mixing-induced, and see whether they tally with the SM predictions. Thus, if the SM amplitude for a particular process be $A_{S M} \exp \left(i \theta_{S M}\right)$ and the BSM amplitude for the same process be $A_{B S M} \exp \left(i \theta_{B S M}\right)$, the total amplitude is given by

$$
A_{t o t} e^{i \theta_{t o t}}=A_{S M} e^{i \theta_{S M}}\left(1+h e^{i \phi}\right)
$$

where $h \equiv A_{B S M} / A_{S M}$ and $\phi=\theta_{B S M}-\theta_{S M}$. The change in CP-asymmetry is essentially governed by $h$, which should be $\sim \mathcal{O}(1)$ for the BSM physics to be visible. Such investigations involve the measurement of the angles as well as the sides of the unitarity triangle (UT). Here, one may face a number of different situations, some of which are:

(i) The three angles of the UT do not sum up to $\pi$. This is a definite signal of new physics, but, considering the errors in determining the angles, may not be easily obtained even in the Bfactories. Even if one measures the functions $\sin 2 \alpha, \sin 2 \beta$ and $\sin ^{2} \gamma$ from CP-asymmetries, one needs to resolve the discrete ambiguity to get the actual values of the angles [3].

(ii) The angles do sum up to $\pi$, but the sides are not in the proper ratio. One needs to determine the sides too for this type of signal.

(iii) CP-asymmetries measured from different modes, which should yield the same angle in SM, give different results. For example, $J / \psi K_{S}$ and $\phi K_{S}$ modes may produce different CP asymmetries (both should give the same angle $\beta$ in $\mathrm{SM}$ ), and one may find nonzero CPasymmetries in $b \rightarrow c$ decay modes of $B_{s}$ (which, in SM, should not give any significant CP-asymmetry).

(iv) One can observe sizable asymmetries in leptonic, semileptonic and radiative B-decays too.

Secondly, one can concentrate on CP-conserving observables. A good place is the branching ratios (BR) of rare modes. CLEO already has some interesting signals [4] which are listed in Table 1; there may be more in near future. Another excellent channel is to look for forbidden modes in the SM (like $B^{+} \rightarrow K^{+} K^{+} \pi^{-}[5]$ ) where even a single event may signal BSM physics. OPAL has looked for such signals and placed limits on BSM couplings [6].

Table 1: Branching ratios $\left(\times 10^{6}\right)$ of the $\eta K$ and $\eta^{\prime} K$ modes. The experimental results are at $2 \sigma$.

\begin{tabular}{|c|c|c|}
\hline Mode & Theoretical BR & Experiment \\
\hline$B^{+} \rightarrow \eta^{\prime} K^{+}$ & $7-41$ & $80_{-9}^{+10} \pm 8$ \\
$B^{0} \rightarrow \eta^{\prime} K^{0}$ & $9-33$ & $88_{-16}^{+18} \pm 9$ \\
$B^{+} \rightarrow \eta K^{*+}$ & $0.03-9$ & $27.3_{-8.2}^{+9.6} \pm 5.0$ \\
$B^{0} \rightarrow \eta K^{* 0}$ & $0.05-3$ & $13.8_{-4.4}^{+5.5} \pm 1.7$ \\
\hline
\end{tabular}

Anyway, we should realize that quantification of BSM physics is something we must approach with caution; qualitative signals $(e . g ., h \sim 1)$ are what we can hope to observe quickly. Of course, if BSM physics is indicated from other experiments, then the B-system can be used to complement and quantify that.

\section{Basic Formalism}

The unitarity triangle probed in B-decays is given by the orthogonality condition

$$
V_{u b} V_{u d}^{*}+V_{c b} V_{c d}^{*}+V_{t b} V_{t d}^{*}=0 .
$$

The triangle, alongwith the angles $\alpha, \beta$ and $\gamma$, is shown in Fig. 1. The only two complex entries in the CKM matrix in Wolfenstein parametrization (WP) are

$$
V_{t d}=\left|V_{t d}\right| \exp (-i \beta), \quad V_{u b}=\left|V_{u b}\right| \exp (-i \gamma) .
$$




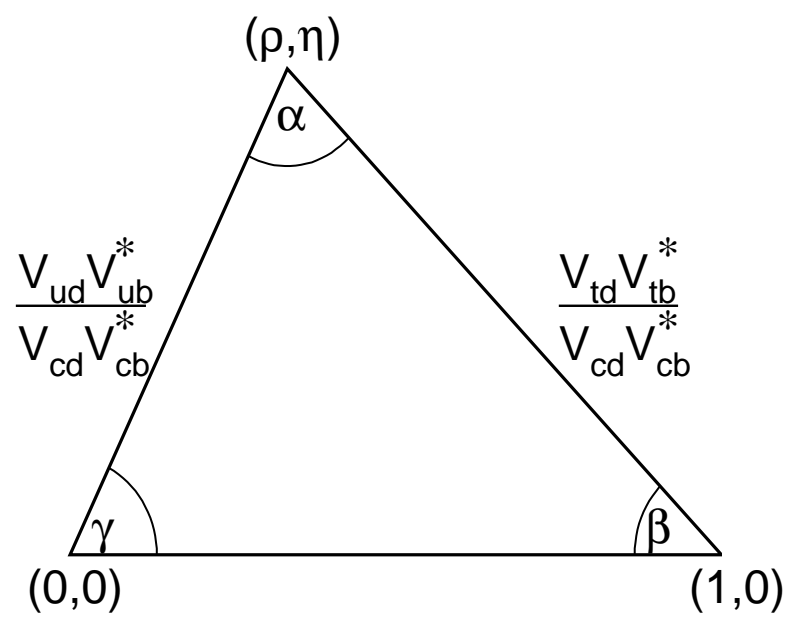

Figure 1: The Unitarity Triangle.

With WP, the tip of the UT has coordinates $(\rho, \eta)$. The central values are [7]

$$
\rho\left(1-\lambda^{2} / 2\right)=0.240_{-0.047}^{+0.057}, \quad \eta\left(1-\lambda^{2} / 2\right)=0.335 \pm 0.042 .
$$

In the SM, the quark-level subprocesses that are important to determine the angles of the UT are shown in Table 2, which is mainly taken from [8]. It is helpful to remember that $B^{0}-\bar{B}^{0}$ mixing measures $2 \beta, b \rightarrow u$ measures $2 \gamma$, presence of both simultameously measures $2 \alpha$ (assuming the UT closes), and $B_{s}-\bar{B}_{s}$ mixing and $b \rightarrow c$ decay are CPconserving to a very good extent. Some of such CP-conserving modes are also shown; a nonzero CP-asymmetry in them (say, in $B_{s} \rightarrow J / \psi \phi$ ) would be an encouraging signal for BSM physics.

Table 2: Quark-level subprocesses for B-decays. P and V denote pseudoscalar and vector mesons respectively.

\begin{tabular}{|c|c|c|c|c|}
\hline No & Quark level & Type & Meson level & Remarks \\
\hline 1 & $b \rightarrow d \bar{u} u$ & $P_{1} P_{2}$ & $\bar{B}^{0} \rightarrow \pi^{+} \pi^{-}$ & $\alpha$ (penguin pollution) \\
\hline 2 & $b \rightarrow d \bar{c} c$ & $P_{1} P_{2}$ & $\overline{B^{0} \rightarrow D^{+} D^{-}}$ & $\beta$ (clean) \\
\hline 3 & $b \rightarrow d \bar{c} c$ & $P V$ & $\bar{B}^{0} \rightarrow J / \psi \pi^{0}$ & $\beta$ (penguin pollution) \\
\hline 4 & $b \rightarrow d \bar{c} c$ & $P V$ & $\bar{B}_{s} \rightarrow J / \psi K_{S}$ & $\lambda^{2} \eta$ (very clean) \\
\hline 5 & $b \rightarrow s \bar{u} u$ & $\overline{P_{1} P_{2}}$ & $B^{0} \rightarrow \pi^{0} K_{S}$ & $\alpha, \gamma$ (not so clean) \\
\hline 6 & $b \rightarrow s \bar{u} u$ & $P_{1} P_{2}$ & $\bar{B}_{s} \rightarrow K^{+} K^{-}$ & $\gamma($ clean $)$ \\
\hline 7 & $b \rightarrow s \bar{c} c$ & $P_{1} P_{2}$ & $\overline{B_{s}} \rightarrow D_{s}^{+} D_{s}^{-}$ & $\lambda^{2} \eta$ (very clean) \\
\hline 8 & $b \rightarrow s \bar{c} c$ & $P V$ & $\bar{B}^{0} \rightarrow J / \psi K_{S}$ & $\beta$ (gold-plated) \\
\hline 9 & $b \rightarrow s \bar{c} c$ & $V_{1} V_{2}$ & $\bar{B}_{s} \rightarrow J / \psi \phi$ & CP-conserving \\
\hline 10 & $\overline{b \rightarrow d \bar{s} s}$ & $\overline{P_{1} P_{2}}$ & $B^{0} \rightarrow K^{0} K^{0}$ & QCD penguin dominates \\
\hline 11 & $b \rightarrow d \bar{s} s$ & $P V$ & $\bar{B}^{0} \rightarrow \pi^{0} \phi$ & EW penguin dominates \\
\hline 12 & $b \rightarrow s \bar{s} s$ & $P V$ & $B^{0} \rightarrow K_{S} \phi$ & $\beta$ (clean) \\
\hline 13 & $b \rightarrow s \overline{d d}$ & $\overline{P_{1} P_{2}}$ & $B_{s} \rightarrow K^{0} K^{0}$ & QCD penguin \\
\hline $14 \mathrm{a}$ & $b \rightarrow u \bar{c} s$ & $\overline{P_{1} P_{2}}$ & $\overline{B^{-} \rightarrow D^{0} K^{-}}$ & $D K$ triangles \\
\hline $14 \mathrm{~b}$ & $b \rightarrow c \bar{u} s$ & $P_{1} P_{2}$ & $B^{-} \rightarrow D^{0} K^{-}$ & measure $\gamma$ \\
\hline
\end{tabular}




\section{Possible New Physics}

In this section, we first briefly review a couple of non-SUSY extensions of the SM, and the results are taken mainly from [10]. After that we discuss two versions of SUSY.

\subsection{Four Generations}

With four quark generations, the CKM matrix is $4 \times 4$, with three independent phases. This makes UT a quadrangle, and the asymmetries measured by different processes will be different from their SM predictions: for example, the asymmetry measured in $B^{0}-\bar{B}^{0}$ mixing is not only $2 \beta$ but some $2\left(\beta+\theta_{d}\right)$ due to the $t^{\prime}$ mediated box.

The smoking gun signals may be the simultaneous measurements of $\alpha, \beta$ and $\gamma$ which will not sum up to $\pi$. Also, $b \rightarrow d \gamma$ and $b \rightarrow d \ell^{+} \ell^{-}$may be enhanced compared to their SM values depending on the magnitudes of $V_{t d}$ and $V_{t^{\prime} d}$ [11]. CP asymmetry in $B \rightarrow J / \psi K_{S}$ is negative for almost half of the parameter space, and almost $40 \%$ of the parameter space predicts the magnitude of $\mathrm{CP}$ asymmetry in $B_{s} \rightarrow J / \psi \phi$ to be more than 0.2 (the SM asymmetry is almost zero) 12 .

\subsection{Multi-Higgs Doublet with no FCNC}

In such models, the CP-asymmetries are almost identical to that of the SM, since the CKM matrix still has the same structure, and $H^{+} \bar{u}_{i} d_{j}$ couplings have the same phase as that of the SM. There may be a significant change in the total amplitude of $B^{0}-\bar{B}^{0}$ mixing due to the $H^{+}$box diagrams, which will in turn affect the value of $V_{t d}$.

An interesting signal in this model may be the $B^{0} \rightarrow \ell^{+} \ell^{-}$rates, which, for some particular choice of the parameter space, can be much higher than the SM ones.

We do not discuss the spontaneous CP-violation scenario, since only spontaneous CPviolation would mean a real CKM matrix, which is ruled out from the $K_{L}-K_{S}$ mass difference and the CDF measurement of $\sin 2 \beta$.

\subsection{Supersymmetry with R-parity Conservation}

The minimal SUSY and its R-parity conserving variants are interesting mainly for the CPviolating observables; any CP-conserving observable like the BRs must have two SUSY particles in the loop and is thereby suppressed in general.

As is well-known, there can be two independent phases in the minimal SUSY which can lead to interesting CP-violating effect. They can be written as

$$
\phi_{A}=\arg \left(A^{*} m_{1 / 2}\right), \quad \phi_{B}=\arg \left(m_{1 / 2} \mu\left(m_{12}^{2}\right)^{*}\right),
$$

where the symbols have their usual meanings. For both these phases $\sim \mathcal{O}(1)$, the dipole moment of neutron, for example, is larger than the experimental limit by two to three orders of magnitude for $100 \mathrm{GeV}$ squarks. This is known as the Supersymmetric CP problem. Another problem arises in the measurement of $\epsilon_{K}$ which turns out to be seven orders of magnitude larger than the experimental number unless there is some fine-tuning among the parameters.

To solve the SUSY flavour problems regarding $\epsilon_{K}$ and dipole moment of neutron, a number of different models were proposed. Among them are: (1) Heavy squarks at the $\mathrm{TeV}$ scale; (2) Universality among right and left squark masses for different generations; (3) Alignment of quark and squark mixing matrices, and (4) Approximate CP-symmetry of the Lagrangian. There are a number of specific flavour models in the literature which incorporates one or more of the above features [13]. As has been pointed out, the effects on measured observables crucially depend on the exact structure of the model, and not all models in a given category have same $\mathrm{CP}$-violating predictions. For example, alignment-type models predict $\sim \mathcal{O}(1)$ contributions to $B^{0}-\bar{B}^{0}$ mixing phases but very small contribution 
to the neutron dipole moment; heavy squark models (for the first two generations) may have a larger $d_{n}$. In Table 3, which is taken from [9], we summarise the predictions of various type of models. For a detailed discussion, see 13 , 9].

Table 3: Prediction of different SUSY flavour models. $\theta_{d}$ is the phase change from the SM prediction $\beta$ in $B^{0}-\bar{B}^{0}$ mixing. a denotes CP-asymmetries for the two decay channels. Taken from [9].

\begin{tabular}{|c|l|c|c|c|}
\hline Model & $d_{n} / d_{n}^{\text {exp }}$ & $\theta_{d}$ & $a_{D^{0} \rightarrow K^{-} \pi^{+}}$ & $a_{K \rightarrow \pi \nu \bar{\nu}}$ \\
\hline SM & $\leq 10^{-6}$ & 0 & 0 & $\mathcal{O}(1)$ \\
Exact & $\leq 10^{-6}$ & 0 & 0 & $\approx \mathrm{SM}$ \\
Universality & & & & \\
Approx. & $\geq 10^{-2}$ & $\mathcal{O}(0.2)$ & 0 & $\approx \mathrm{SM}$ \\
Universality & & & & \\
Approx. CP & $\sim 10^{-1}$ & $-\beta$ & $\mathcal{O}\left(10^{-3}\right)$ & $\mathcal{O}\left(10^{-5}\right)$ \\
Alignment & $\geq 10^{-3}$ & $\mathcal{O}(0.2)$ & $\mathcal{O}(1)$ & $\approx \mathrm{SM}$ \\
Heavy $\tilde{q}$ & $\approx 10^{-1}$ & $\mathcal{O}(1)$ & $\mathcal{O}\left(10^{-2}\right)$ & $\approx \mathrm{SM}$ \\
\hline
\end{tabular}

Another interesting observable is the forward-backward lepton asymmetry (as well as the absolute BRs) in $B \rightarrow X_{s} \ell^{+} \ell^{-}$where $\ell=e$ or $\mu$ [14. For both the leptons, the SM predictions for $A_{F B}$ is 0.23 but it can vary from 0.33 to -0.18 in SUSY models. The negative $A_{F B}$ constitutes an interesting signal. The BRs can be enhanced by a factor of four or can be suppressed by a factor of two, which should also be measured in the B-factories.

\subsection{Supersymmetry without R-parity}

R-parity violating (RPV) SUSY has one great advantage over the non-RPV SUSY models: the new physics contributions appear in the tree-level, and hence can greatly enhance or suppress the SM contributions. Here we will discuss the popular approach, i.e., we will consider all RPV couplings to be free parameters, constrained only by various experimental data, and study its consequences on B-decays.

The physics of RPV has been discussed in detail in the literature, and here we will only quote the main results. For $B \rightarrow M_{1} M_{2}$ decays ( $M$ is any meson in general) the relevant pair of couplings is either $\lambda^{\prime} \lambda^{\prime}$ or $\lambda^{\prime \prime} \lambda^{\prime \prime}$ type (but not both simultaneously). For $B \rightarrow M \ell^{+} \ell^{\prime-}$ decays, it is $\lambda \lambda^{\prime}$ and $\lambda^{\prime} \lambda^{\prime}$ together. For example, we can have sneutrino/squark mediated $b \rightarrow d_{i} \bar{d}_{j} d_{k}$ decays and selectron/squark mediated $b \rightarrow d_{i} \bar{u}_{j} u_{k}$ decays $(i, j, k$ are generation indices). All B-decay modes are affected by suitable pair of RPV couplings; more specifically, all UT angles can change from their SM predictions. In SM, the decays $B \rightarrow J / \psi K_{S}$ and $B \rightarrow \phi K_{S}$ measure the same angle $\beta$; with RPV, the measured CP-asymmetries may be different, which will definitely signal new physics [15]. One can see forbidden modes like $B^{+} \rightarrow K^{+} K^{+} \pi^{-}$originating from the SM forbidden $b \rightarrow s s \bar{d}$ decay [5]. CP-asymmetries $\sim 100 \%$ in the measurement of the UT angle $\gamma$ can be obtained even from $B^{+}$decays [16]; thus, study of $B^{+} \mathrm{s}$, alongwith $B^{0} \mathrm{~s}$ and $B_{s} \mathrm{~s}$, are of paramount importance. The leptonic forward-backward asymmetries are modified too: for a pure $\lambda \lambda^{\prime}$ type coupling, there is no FB asymmetry, whereas for a $\lambda^{\prime} \lambda^{\prime}$ type coupling, it is in the opposite direction from SM [17].

Another important feature is that RPV couplings can enhance or suppress the BRs significantly. As we have seen, the CLEO data on $\eta^{\prime} K$ and $\eta K^{*}$ are quite far away from the SM prediction. It has been shown 18 that a moderate value of the product coupling $d_{222}^{R} \equiv$ $\lambda_{i 23}^{\prime} \lambda_{i 22}^{\prime}$ (each $\lambda^{\prime}=0.05-0.09$, say), perfectly compatible with the experimental bounds, can enhance the BRs to their experimental value. This is demonstrated in Fig. 2 where we have plotted the BR against $\xi=1 / N_{c}^{e f f}$, using $m_{s}(1 \mathrm{GeV})=165 \mathrm{MeV}$. At the same time, this product suppresses decays like $B^{0} \rightarrow \phi K$; in SM, this decay is allowed only for $\xi<0.23$ but with RPV, larger ranges of $\xi$ are allowed. 


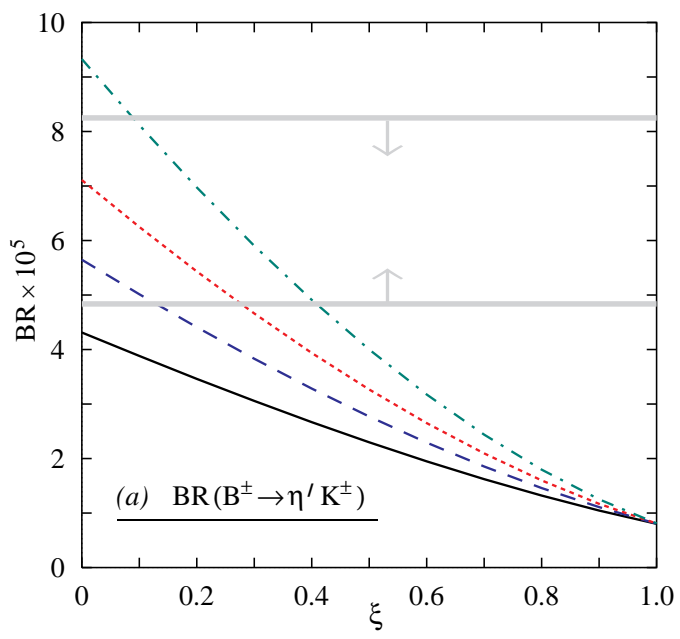

Figure 2: $\mathrm{BR}$ for $B \rightarrow \eta^{\prime} K$ as a function of $\xi$. The solid curve gives the $\mathrm{SM}$ value. In the presence of a $\lambda_{i 23}^{\prime} \lambda_{i 22}^{\prime}$ operator with $m_{\tilde{\nu}}=200 \mathrm{GeV}$, the long-dashed, short-dashed and dot-dashed curves correspond to the cases where each of the two $\lambda^{\prime}$ s equal $0.05,0.07$ and 0.09 respectively. The thick lines correspond to the experimental bounds.

However, the SM range is in conflict with other PV modes such as $B^{ \pm} \rightarrow \omega K^{ \pm}$and $B^{ \pm} \rightarrow \omega \pi^{ \pm}$. The former requires either $\xi<0.05$ or $0.65<\xi<0.85$ while the latter requires $0.45<\xi<0.85[19]$. Interestingly, the $d_{222}^{R}$ operator affects $B^{ \pm} \rightarrow \phi K^{ \pm}$while the other two decay modes are blind to it. This additional contribution interferes destructively with the SM amplitude and $B R\left(B^{ \pm} \rightarrow \phi K^{ \pm}\right)$is suppressed leading to a wider allowed range for $\xi$. For example, with each $\lambda^{\prime}=0.09, \xi$ can be as large as 0.8 , thus allowing for a common fit to all the three $(P V)$ modes under discussion?. (Note that $\lambda^{\prime}=0.09$ is just ruled out from $B \rightarrow X_{s} \nu \bar{\nu}$ data 20, 21] if we assume a sneutrino-squark degeneracy; but there is every reason to believe that squarks are heavier than sneutrinos, in which case our analysis still stands.) $d_{222}^{R}$ also affects $V V$ decay modes such as $\left(B \rightarrow \phi K^{*}\right)$. As this calculation involves a few more model dependent parameters, we do not discuss it here.

\section{Conclusions}

The study of B-decays, both in the CP-conserving and CP-violating fronts, is quite interesting to study indirect effects of new physics, more so in view of the upcoming B-factories. CLEO has already given some food for thought. Among various new physics models, nonSUSY extensions of the SM mainly affect the $B^{0}-\bar{B}^{0}$ amplitude, and, maybe, phase. The determination of $V_{t d}$ may be affected too. Different SUSY flavour models will have different signatures regarding the neutron dipole moment and asymmetries in $J / \psi K_{S}$ and $\pi \nu \bar{\nu}$ modes. RPV SUSY models can contribute to almost all B-decays, and can even induce some $\mathrm{SM}$ forbidden decyas. One of the important achievements is to explain the CLEO result on $B \rightarrow \eta^{\prime} K$ with RPV.

However, this is only about the observation of BSM physics, and to have a qualitative measurement, one needs to minimize the theoretical errors, which will be the biggest challenge to the theoreticians in the next few years. In short, we await some really exciting years on both theoretical and experimental fronts!

\footnotetext{
${ }^{2}$ Note that the favoured value of $\xi$ for the $P P$ and $P V$ modes still continue to be different. While this is not a discrepancy, a common $\xi$ for both these sets can be accommodated for values of $\lambda^{\prime}$ slightly larger than that we have considered.
} 


\section{Acknowledgements}

I thank Rahul Basu and other organisers of WHEPP-6 for providing a most stimulating atmosphere. Some of the material presented here is based on the work done with Debajyoti Choudhury and Bhaskar Dutta.

\section{References}

[1] See, e.g., Y. Grossman and M.P. Worah, Phys. Lett. B395, 241 (1997); M. Gronau and D. London, Phys. Rev. D55, 2845 (1997).

[2] For a discussion of such uncertainties, see, e.g., H. Quinn, hep-ph/9912325.

[3] B. Kayser and D. London, hep-ph/9909560.

[4] CLEO collaboration, hep-ex/9908019, hep-ex/9912059.

[5] K. Huitu et al, Phys. Rev. Lett. 81, 4313 (1998).

[6] OPAL collaboration, hep-ex/0002008.

[7] F. Caravaglios et al, hep-ph/0002171.

[8] A.J. Buras and R. Fleischer, in Heavy Flavours II, A.J. Buras and M. Lindner (eds.) World Scientific, Singapore (1997).

[9] Y. Nir, hep-ph/9911321.

[10] M. Gronau and D. London, in [1].

[11] W.-S. Hou, A. Soni and H. Steger, Phys. Lett. B192, 441 (1987).

[12] D. London, Phys. Lett. B234, 354 (1990).

[13] G. Barenboim and M. Raidal, Phys. Lett. B457, 109 (1999), and references therein.

[14] E. Lunghi and I. Scimemi, hep-ph/9912430.

[15] D. Guetta, Phys. Rev. D58, 116008 (1998).

[16] G. Bhattacharyya and A. Datta, Phys. Rev. Lett. 83, 2300 (1999).

[17] J.-H. Jang, Y.G. Kim and J.S. Lee, Phys. Lett. B408, 367 (1997).

[18] D. Choudhury, B. Dutta and A. Kundu, Phys. Lett. B456, 185 (1999).

[19] N.G. Deshpande, B. Dutta and S. Oh, hep-ph/9712445.

[20] Y. Grossman, Z. Ligeti and E. Nardi, Nucl. Phys. B465, 369 (1996); ibid 480, 753 (1996) (E).

[21] ALEPH collaboration, in ICHEP '96. Proceedings of the 28th Int. Conference on HEP. Warsaw, Z. Ajduk and A. Wroblewski (eds.) World Scientific, Singapore, (1997). 